\title{
Experimental Study on Phase Conjecture Based on Two-dimensional Image and Carrying Three- dimensional Image With Mist in Electro-holographic Reconstruction *
}

\section{ZHAO Zhi-xiong (D 694881468@qq.com)}

Institution of electrical and electronic Engineering https://orcid.org/0000-0002-7608-9238

\section{ZHANG Hua}

Chongqing Vocational and Technical University of Mechatronics

\section{Kuang Qing-yun}

Chongqing Vocational and Technical University of Mechatronics

\section{Li Bo}

Chongqing Vocational and Technical University of Mechatronics

Hu Lin

Chongqing Vocational and Technical University of Mechatronics

\section{Research Article}

Keywords: phase conjecture, two-dimensional(2D) image, three-dimensional(3D), electro-holographic reconstruction, mist

Posted Date: January 11th, 2022

DOI: https://doi.org/10.21203/rs.3.rs-1067466/v1

License: (c) (i) This work is licensed under a Creative Commons Attribution 4.0 International License.

Read Full License 


\title{
Experimental study on phase conjecture based on two-dimensional image and carrying three-dimensional image with mist in electro-holographic reconstruction *
}

\author{
ZHAO Zhi-xiong ${ }^{1 * *}$, ZHANG Hua ${ }^{1}$, Kuang Qing-yun ${ }^{1}$, Li Bo ${ }^{1}$, Hu Lin ${ }^{2}$ \\ 1.School of Electrical and Electronic Engineering, Chongqing Vocational and Technical University of Mechatronics, \\ Chong Qing, China. \\ 2.Chongqing Bishan Midlle School \\ (Received XXXXX; Revised XXXXX)
}

\begin{abstract}
A method is proposed for phase conjecture based on the intensity curve of a two-dimensional(2D) image by computing a polynomial equation. The intensity values of the 2D image, which represents the distance between the image detectors and the three-dimensional(3D) scene is converted to phase information by our method. The results of numerical calculation with phase conjecture are analyzed. And what's more, the numerical reconstruction results with phase information obtained as initial phase factors of a complex object for Fresnel kinoform and dynamic pseudorandom-phase tomographic computer holography(DPP-TCH) are compared. The peak signal-to-noise ratio(PSNR) and correlation coefficient (CC) between the reconstructed images and original object are analyzed. An experimental system is designed for photoelectric holographic reconstruction based on phase-only liquid crystal spatial light modulator(LC-SLM) and mist screen. The electro-optical experimental results indicate that suppressed the speckle noise 3D images that can be observed with naked eye have been obtained.
\end{abstract}

Document code: A Article ID: XXXX-XXXX(XXXX)XX-XXXX-X

DOI XX.XXXX/XXXXXX-XXX-XXXX-X

\footnotetext{
* This work has been supported by the Science and Technology Research Program of Chongqing Municipal Education Commission (Grant No.KJQN202103703), by the Science and Technology Research Program of Chongqing Municipal Education Commission (Grant No.KJZD-K201903701).

** E-mail: 694881468@qq.com
} 
Holography is an especially excellent form of display, because unlike other imaging technologies, it stores 3D information of an object, including amplitude and phase[1]. Computer-generated holography is widely used in holography displays, and hence, computer holography and digital holography have been investigated extensively[2]. Computer generated holography can enable not only generation of holograms of photographs conveniently and efficiently with different coding methods, but also fabricating holograms of objects that do not exist or virtual objects through different object function descriptions. In addition, no optical setups are required for computer holography, thus eliminating optical lens error and environmental influences. Therefore, computer-generated holograms(CGHs) have low noise and higher repeatability. Owing to these advantages, CGHs can simulate spatial filters and transform components and can be satisfactorily applied to holographic 3D virtual displays[3-5]. Either the amplitude and phase information, or just the phase information on the hologram plane is loaded on a SLM to reconstruct the $3 \mathrm{D}$ diffusive objects.

Image detectors can only gather the intensity information of a light field and cannot directly collect phase information, which contributes to $\sim 75 \%$ of the information regarding the objects[6], because the oscillation frequency of the light field is relatively high. In studies on phase retrieval(PR), which employ the diffraction of the light field as the model of numerical simulation, the intensity distribution of the emergent plane is obtained by computing the diffraction process of the assumed incident plane, meanwhile, acquiring the final phase distribution of the intensity information that best matches the real scene by comparing the intensity data produced by the real phase with the intensity of the light field emergent plane is research hotspot. R.W.Gerchberg and W.O.Saxton first proposed the concept phase retrieval to solve the problem of electron microscopy imaging[7,8]. The result of this algorithm is not very accurate.In order to solve the above problems, many new algorithms based on the Gerchberg-Saxton(GS) PR iteration algorithm were proposed. For example, $\mathrm{Gu}$ et al.[9] developed an algorithm using the weighted phase as initial phase data; this algorithm has better accuracy, but there is a trade-off with the rate of convergence. Huang et al.[10] proposed a method to solve this problem. The PR method which is proposed by Guo Yiming[11] is a fast, high precision and method of effective phase retrieval.

The CGHs of 3D objects can be computed by numerous techniques, such as the tomographic approach[12,17], Fresnel zone approach[13], and multi-view image synthesizing approach[14]. The tomographic method often divides the 3D objects into a series of planar segments. An initial random phase is employed as the phase distribution of the complex amplitude for this effectively allotting the information content over the entire region occupied by the hologram plane.

In this paper, we propose a new method for phase conjecture based on the intensity information of $2 \mathrm{D}$ images from 3D scenes by fitting a polynomialm, This polynomial is computed by a simple and fast procedure that is already available. The method is based on the gray values along line profiles and phase information profile hidden in real $2 \mathrm{D}$ pictures. The phase data obtained based on the 2D images by our method form the phase part of the complex amplitude of the target. Meanwhile, the gray values of the 2D images indicate the amplitude. Thus, complex amplitude is obtained. Instead of Burch encoding, which has low diffraction efficiency[15], kinoform encoding is employed to generate the $\mathrm{CGH}$. Kinoform encoding can adequately eradicate the conjugated images[15], which may severely affect the quality of reconstructed images. The results of numerical calculation and photoelectric via the above mentioned method are analyzed.

Grayscale images whose gray value represent the profiles of phase and gray gradient change turn into phase variation are gleaned from Optoelectronic components $\mathrm{CCD}$ (or CMOS) and 3D scene are placed horizontally. 2D intensity image is collected from 3D object which is placed vertically in front of images detectors, light energy distribution function of scene is calculated according to the light energy in CCD. Gray values of every pixel representative the distance between $3 \mathrm{D}$ object and $\mathrm{CCD}$. The phase information is calculated by fitting the gray curve obtained by transforming the different positions of two perpendicular line in $2 \mathrm{D}$ intensity image. The continuous phase is quantized to 0 and $2 \pi$ according to the gray gradient values. The phase conjecture procedure is vividly illustrated by Fig.1. The procedure can be written as:

$$
\begin{aligned}
& x_{h}=a_{0}+a_{1} x+a_{2} x^{2} \\
& y_{v}=b_{0}+b_{1} y+b_{2} y^{2}
\end{aligned}
$$

as the two curves fitted along the horizontal and vertical grayscale images, respectively. where $x_{h}$ and $y_{v}$ is horizontal and vertical the phase information. The phase distribution becomes ultimately:

$$
\varphi(m, n)=\exp \left[i\left(a_{1} m+b_{1} n+a_{2}{ }^{2} m^{2}+b_{2}{ }^{2} n^{2}\right)\right]
$$

Where $m$ and $n$ define the sampling number.

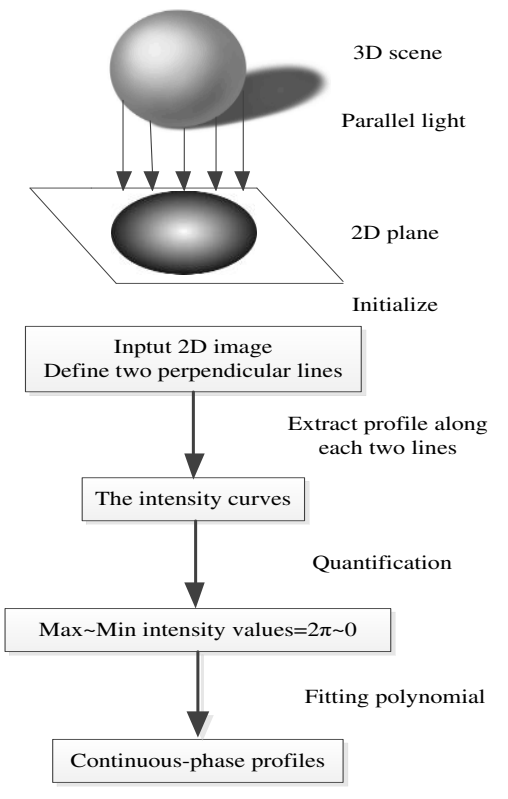

Fig.1 Flow of phase conjecture

The core process of GS algorithm for phase retrieval is to 
obtain the phase distribution of hologram plane by iterative operations of multiple Fourier transforms and inverse Fourier transformations[16]. The basic process of GS algorithm for phase retrieval is shown in Fig.2.

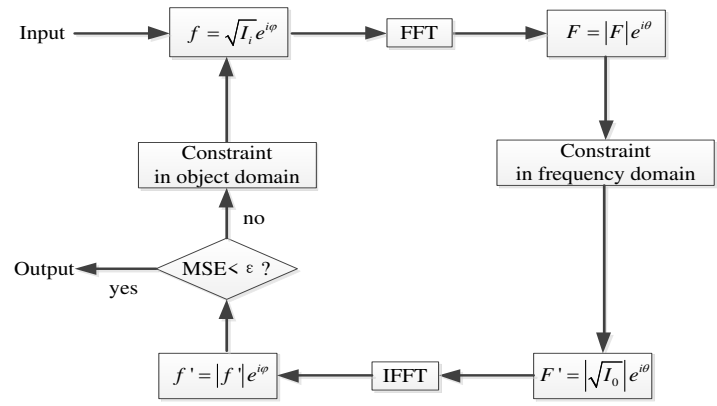

Fig.2 Block diagram of GS algorithm[16]

In the Fig.2, $\sqrt{I}_{i}$ and $\sqrt{I}_{o}$ is the amplitude of input plane and output plane, respectively. $\varphi$ is random phase, or estimated value to dwindle iteration error of initial phase. when normalized mean-square error is lessen than set value. MSE can be written as[17]:

$$
M S E=\frac{1}{M N} \sum_{m=1}^{M} \sum_{n=N / 2+1}^{N}\left(|f(m, n)|^{2}-\left|f^{\prime}(m, n)\right|^{2}\right)^{2}
$$

where $\mathrm{M}$ and $\mathrm{N}$ respectively as the total numbers of sampling unit in the direction of $\mathrm{x}$ and $\mathrm{y}$.

Fig. 3 shows geometry system used to compute the CGH of a series of planar segments. The Fresnel diffraction of each segment was calculated by using the single-step Fresnel(SSF) diffraction method - Fourier transform algorithm[16]. The contribution of object plane to the hologram plane can be expressed as:

$$
\begin{aligned}
H_{i}(\varepsilon, \eta)= & \frac{\exp \left(j 2 \pi d_{i} / \lambda\right)}{j \lambda d_{i}} \exp \left[\frac{j \pi\left(\varepsilon^{2}+\eta^{2}\right)}{\lambda d_{i}}\right] \times \\
& \iint\left\{O_{i}\left(x_{i}, y_{i}\right) \exp \left[j \varphi_{i}\left(x_{i}, y_{i}\right)\right] \times\right. \\
& \exp \left[j \varphi_{i}\left(x_{i}, y_{i}\right) \exp \left[\frac{j \pi\left(x_{i}^{2}+y_{i}^{2}\right)}{\lambda d_{i}}\right] \times\right. \\
& \left.\exp \left(-j 2 \pi \frac{x_{i} \varepsilon+y_{i} \eta}{\lambda d_{i}}\right)\right\} d x d y
\end{aligned}
$$

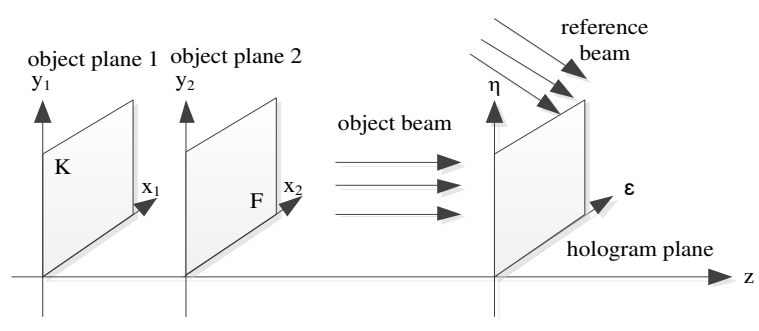

Fig.3 Schematic diagram of computer generated tomography

where $(\varepsilon, \eta)$ indicates holographic coordinate, $\left(x_{i}, y_{i}\right)$ represent the coordinate of the $i_{\text {th }}$ segment, $\varphi_{i}\left(x_{i}, y_{i}\right)$ which used to reduce the dynamic range of the object wave spectrum provides the random phase information. The optical distribution from all segments are superimposed on the hologram plane as:

$$
H(\varepsilon, \eta)=\sum_{i=1}^{n} H_{i}(\varepsilon, \eta)=A(\varepsilon, \eta) \exp [j \varphi(\varepsilon, \eta)]
$$

where $A(\varepsilon, \eta)$ provides the amplitude of the hologram, $\varphi(\varepsilon, \eta)$ is the diffusive phase.

Complex amplitude distribution of the $3 \mathrm{D}$ scene on the hologram plane can be obtained by tomographic approach mentioned above. In the process of reconstruction, hologram obtained through above method is multiplied by the conjugate reference beam, and then the hologram perform inverse diffract by the Fresnel diffraction formula, at last the reconstructed image at different distances can be obtained.

The phase conjecture process is illustrated by simulated experiments below. A 2D intensity image extracted from 3D sphere is showed as Fig.4(a). Its radius is $0.158 \mathrm{um}$. Two perpendicular lines is defined in Fig.4(a), one for each direction $\mathrm{m}$ and $\mathrm{n}$, which are, respectively, the horizontal and vertical directions. Along each of two lines, two gray values lines appear in Fig.4(b) and Fig.4(c), and according to Eq. (1), the phase distribution can be obtained easily, as shown in Fig.4(d).

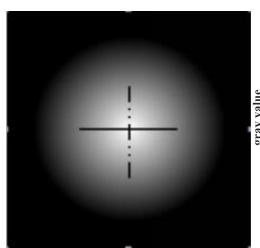

(a)

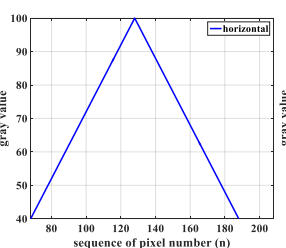

(b)

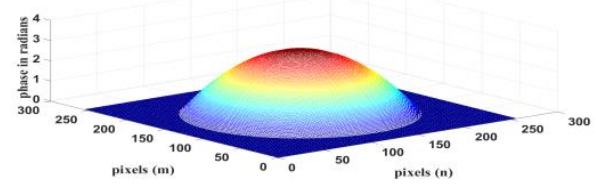

(d)
Fig.4 The phase conjecture. (a) 2D intensity image; (b) Intensity cure by horizontal line in (a); (c) Intensity cure by vertical line in (a); (d) Phase distribution by fitting polynomial equation.

The experimental results show that partial phase distribution of $3 \mathrm{D}$ objects can be obtained from $2 \mathrm{D}$ images. It is noting that phase conjecture method is applied to conjecture the phase distribution of projection part, while the phase of other perspective $2 \mathrm{D}$ images is not valid.

According to the Fig.2, the result of phase retrieval is shown in Fig.5(a). When the number of iteration reaches 100, the MSE variation according to iterate times is shown in Fig.5(b).

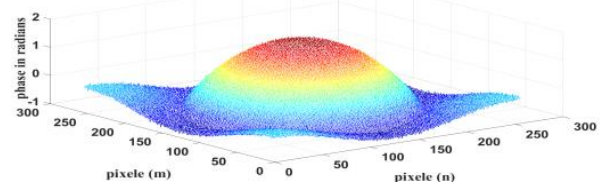

(a) 


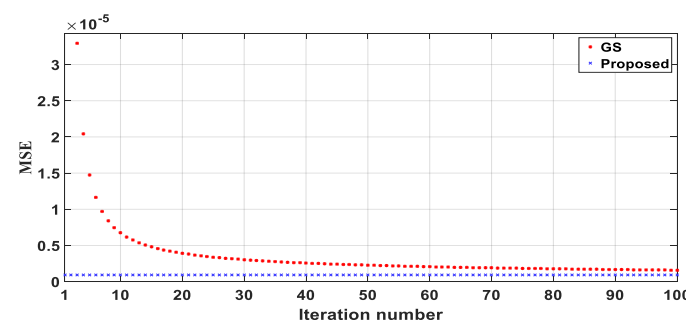

(b)

Fig.5 GS phase retrieval. (a) the result of GS algorithm. (b) MSE variation according to iterative number

According to the results of the two simulated experiments, it is not difficult to conclude that the phase distribution by proposed methd is more close to the actual phase than the result of GS algorithm, which require multiple iterative computation. Difference between the retrieved result and the given phase by different method are shown in Fig.6(a) and Fig.6(b) respectively. MSE values of proposed method is $9.327 \times 10^{-7}$, which is much less than $1.5822 \times 10^{-6}$ of GS requires several operations. Not only the complexity the calculation but also the time required for proposed method are less than that needed for GS algorithm according the Fig.5(b).

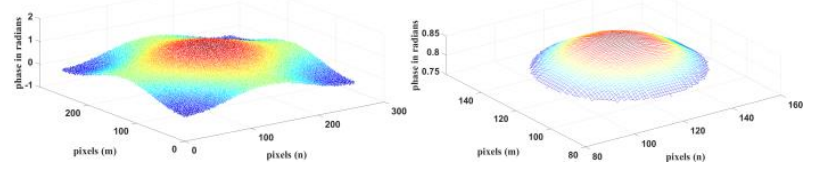

Fig.6 Difference between retrieval result and given phase. (a) GS algorithm. (b) proposed method.

Fig.4(a) as the amplitude and Fig.4(d) as the phase of original complex amplitude distribution respectively. So complex amplitude distribution in hologram plane can be obtained by simulating the optical transmission process, and the Fresnel kinoform contributed by the wavefront is shown in Fig.7(a). The intensity and phase distribution of reconstructed images at $350 \mathrm{~mm}$ are shown in Fig.7(b) and Fig.7(c).

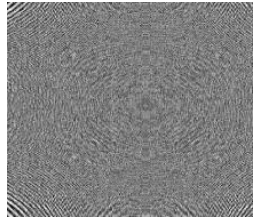

(a)

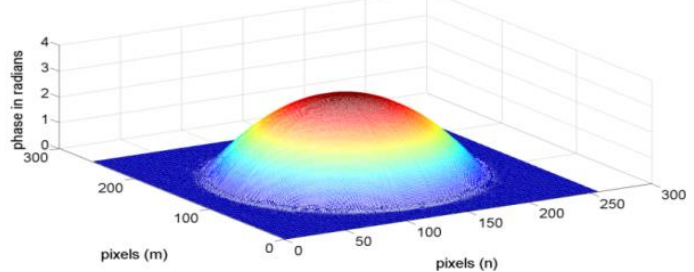

(c)

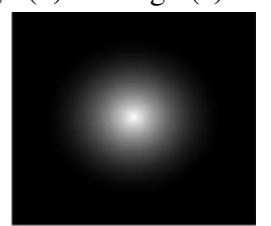

(b) inte

Fig.7 (a) Fresnel kinoform. (b) intensity distribution of reconstruction(c) phase distribution of reconstruction distance is $50 \mathrm{~mm}$ is demonstrated DPP-TCH method. The original 3D object and kinoform are shown in Fig.8. The distance between the letter $\mathrm{K}$ and hologram is $400 \mathrm{~mm}$, and the distance between the letter $\mathrm{F}$ and hologram is $350 \mathrm{~mm}$. Superposed results of 200 reconstructed images at 400 and 350 $\mathrm{mm}$ are shown in Fig.8(c) and Fig.8(d) respectively.

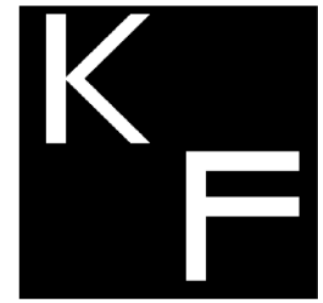

(a)

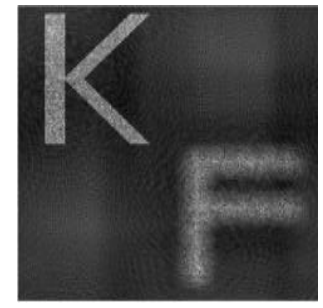

(c)

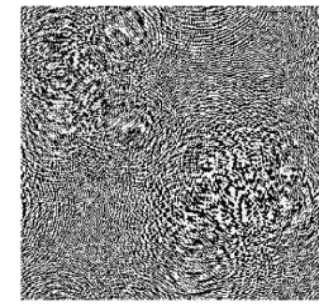

(b)

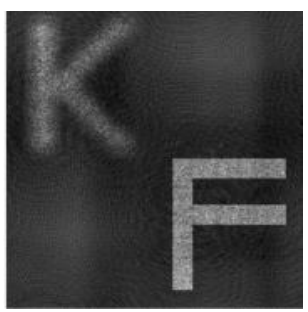

(d)
Fig.8(a) Original 3D image. (b) kinoform.(c) 200 reconstructed images at $400 \mathrm{~mm}$. (d) 200 reconstructed images at $350 \mathrm{~mm}$.

As shown in Fig.7(c), reconstructed phase distribution which contains 3D topography information of 3D objects is display, apparently, the reconstructed phase is not only close to initial phase but also its radian is same to initial value. However, DPP-TCH as a kind of technology for displaying 3D objects, it is worth that not all reconstructed image planes are focus, when focusing on the letter $\mathrm{K}$, the letter $\mathrm{F}$ would blur, and vice versa. In order to evaluate the qualities of reconstructed images, CC and PSNR between reconstructed images and original images are utilized. CC and PSNR[17] are defined respectively as follows:

$$
\begin{gathered}
C C=\frac{\operatorname{Cov}\left(I_{1}, I_{2}\right)}{\sqrt{\operatorname{Var}\left(I_{1}\right) \cdot \operatorname{Var}\left(I_{2}\right)}} \\
P S N R=10 \lg \left(\frac{\sum_{i=1}^{M} \sum_{j=1}^{N}\left[I_{1}(i, j)\right]^{2}}{\sum_{i=1}^{M} \sum_{j=1}^{N}\left[I_{1}(i, j)-I_{2}(i, j)\right]^{2}}\right)
\end{gathered}
$$

where $\operatorname{Cov}\left(I_{1}, I_{2}\right)$ indicates covariance operation between $I_{1}$ and $I_{2}, \operatorname{Var}\left(I_{1}\right)$ is the variance values of $I_{1}(i, j)$, $I_{1}(i, j)$ and $I_{2}(i, j)$ are original and reconstructed image(including phase information) correspondingly.

$\mathrm{CC}$ and PSNR curves of the part-images $(\mathrm{K})$ in the reconstructed image at $400 \mathrm{~mm}$ from different number of kinoform are shown in Fig.9(a) and (b).

A 3D object composed of two planar segments whose 


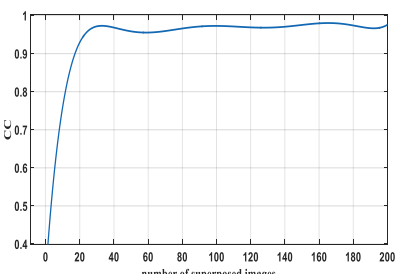

(a)

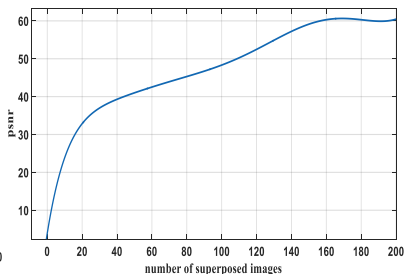

(b)
Fig.9( a) CC . (b) PSNR variation curve according to superposing number of reconstructed part-images at $400 \mathrm{~mm}$.

The CC and PSNR values of proposed method and DPP-TCH are shown in following table. According to Tab.1, through comparison with the 200 superposed reconstructed part-image at $400 \mathrm{~mm}$, values of CC and PSNR increase to 0.9866 and 82.8665, respectively. And the elapsed time of DPP-TCH is 482 seconds $=2.41 \times 200$, however the reconstructed image including real phase information could be obtained by single diffraction of which elapsed time is only 3.27 seconds. The simulated experiment results show that reconstructed phase distribution encompass actual 3D topography information is acquired and speckle noise can be almost removed.

\section{Tab.1 The comparative CC and PSNR values of different method}

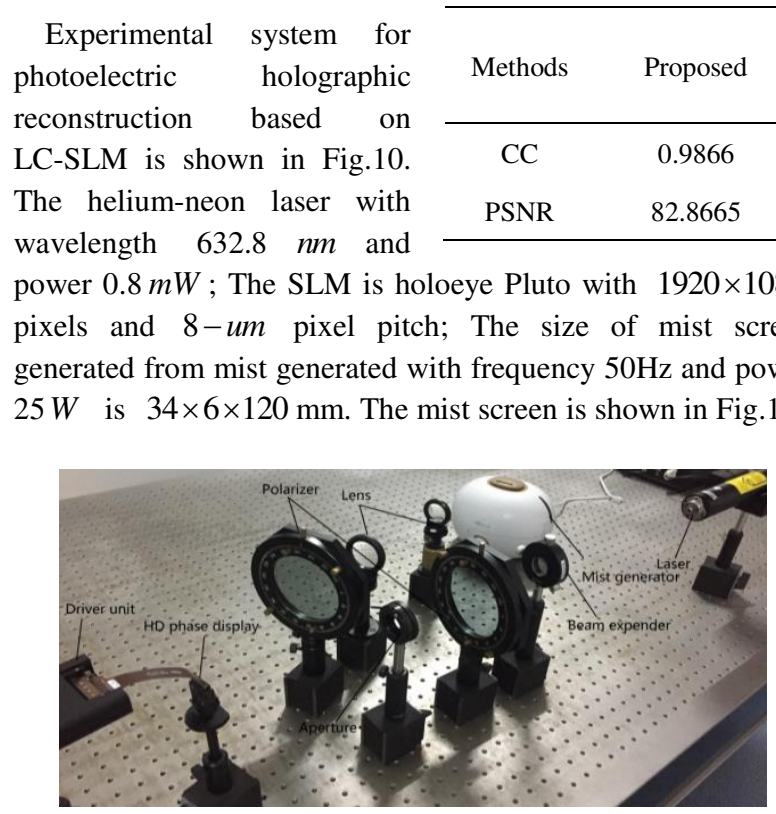

Fig.10 Optical setup for holographic display

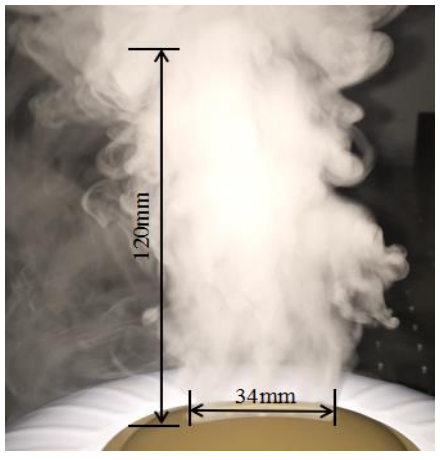

Fig.11 Mist screen

The incident illumination light which is turn into polarized and parallel light by polarizer and beam expender, respectively, is phase modulated by kinoform loading on the SLM. Fig. 12 is the electro-optical reconstructed images which is captured by $\mathrm{CCD}$ at $300 \mathrm{~mm}$. It is obvious that the electro-optical reconstruction image carried with cubic mist screen is display in air.

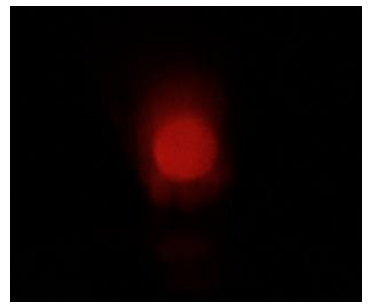

Relative value $(\%)$

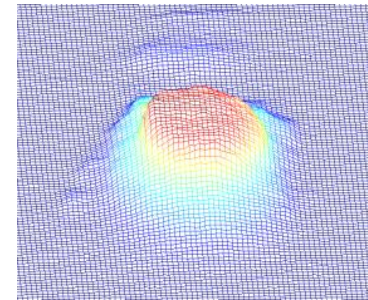

(a) (b)

Fig.12 3D reconstructed image; (a) 3D image carried with mist screen in optoelectronic reconstruction; (b) 3D intensity distribution

A novel method is proposed for phase conjecture. In this method, the 2D projected image is used to calculate the values of involved parameters by using the phase distribution as the initial phase factors in the object domain. For this purpose, a fitting curve extracted along the profiles defined by two perpendicular lines is used. Unlike the traditional phase retrieval method, the proposed phase conjecture method is simple and efficient. It has been validated as well. No complicated diffraction optical path or iterative operations are involve in this method. The digital experiment results show that the phase distribution of a 3D object can be obtained easily. The results of numerical reconstruction achieved by using phase information as the initial phase factors of a complex object for Fresnel kinoform and DPP-TCH are compared. The values of CC and PSNR achieved by the proposed method are higher than those for DPP-TCH, the time required for the proposed method is significantly less than that needed for DPP-TCH. Finally, the elecctro-optical reconstruction generated by the 3D electro-optical holographic display system 
was studied. However, owing to imperfections attributed to airflow, wind, and inhomogeneous distribution of mist, and because the peak height of the reconstructed form is only 0.158 um, the depth cues cannot be detected easily. Future work will aim to improve the $3 \mathrm{D}$ reconstructed images.

\section{References}

[1] Malek M, Allano D, Coëtmellec S, et al, Optics Express 12(10), 2270-9 (2014).

[2] Nagashima K, Optics \& Laser Technology 35(3), 181-186 (2003).

[3] Frère C, Leseberg D, Applied Optics 28(12), 2422-5 (1989).

[4] Schwerdtner A, Haussler R, Leister N, US B2, 7643189 (2010).

[5] Schwerdtner A, Haussler R, Leister N, US B2[P], 7400431 (2008).

[6] M Mir, B Bhaduri, R Wang,et al, Progress in Optics 57,133-217 (2012).
[7] Gerchberg R W, Saxton W O, Optik 34(3), 275-+(1971).

[8] Gerchberg R W, Optik, 35, 237-250 (1972).

[9] Xiang G U, Ke-Shu X U, Journal of Fudan University, 2000.

[10] Huang Lixin, Yao Xin, Cai Dongmei, et al, Chinese Journal of Lasers 37, 1218-1221 (2010).

[11] Guo Y, Zhang F, Song Q, et al, Acta Optica Sinica 36(9), 0912001 (2016).

[12] Wang Z, Jia J, Zhu M, et al, Synthetic Metals 222 (2016).

[13] Zhang X, Liu X, Chen X, SPIE 5636, 109-115 (2005).

[14] Abookasis D, Rosen J, Journal of the Optical Society of America 20, 1537-45 (2003).

[15] Zhixiong Zhao, Hai Liu, Yunxiu Shui, Lin Hu, Y.Yang, SPIE 10255, 1025503 (2017).

[16] J W Goodman, Englewood:Roberts \& Co.Publishers (2005).

[17] Zeng Z, Zheng H, Lu X, et al, Optical Review 22, 853-861 (2015). 